ANÁLISIS SOCIOECONÓMICO Y POTENCIALIDADES ECOTURÍSTICAS EN LA CIÉNAGA LA CAIMANERA ${ }^{1}$

\title{
SOCIOECONOMIC ANALYSIS AND ECOTURISMO POTENTIAL IN THE SLEW CAIMANERA
}

\author{
Carlos Buelvas Meza $^{72}$, Sandra Rojas Sevilla ${ }^{3}$ y Ulises Tinoco Cantillo ${ }^{4}$ \\ Corporación Universitaria del Caribe-(Cecar), Colombia
}

RECIBIDO: 21 de septiembre de 2014

ACEPTADO: 16 de noviembre de 2014

\section{RESUMEN}

El presente artículo es producto de la investigación Ciénaga la Caimanera: un mundo de oportunidades para Coveñas a través del ecoturismo, que fijó como objetivo realizar un análisis socioeconómico de los hogares de ese lugar de aquel municipio, en el departamento de Sucre (Colombia), así como del potencial ecoturístico. Basándose en el paradigma empírico-analítico, con enfoque cuantitativo, su diseño es no experimental de tipo aplicado y nivel descriptivo; la población la conformaron 137 hogares, la muestra fue de 98 hogares, y el proceso de muestreo fue probabilístico (muestreo aleatorio simple). Las técnicas de investigación utilizadas fueron: la encuesta y la observación directa. Los resultados obtenidos evidenciaron falta de infraestructura física, con la consiguiente insatisfacción de necesidades en materia de educación, salud, vivienda, agua potable, saneamiento básico; adicionalmente, hay una oferta de trabajo con escasas posibilidades de insertarse en el mercado laboral, pese al alto potencial ecoturístico que representa el sistema local de humedales: caños, arroyos y ciénagas, entre los que se destaca la cuenca de la ciénaga la Caimanera, con un área de 430 hectáreas. La insuficiencia y deficiencia de infraestructura de servicios públicos dificulta el aprovechamiento del potencial ecoturístico como medio de desarrollo de la comunidad.

Palabras clave: Ecoturismo, comunidades, cultura, infraestructura.

\begin{abstract}
This article is the result of the investigation "Swamp Caimanera: a world of opportunities through ecotourism Coveñas" whose objective was to conduct a socio-economic analysis of households in the Swamp Caimanera Coveñas municipality, department of Sucre Colombia, and ecotourism potential. It is based on the analytical empirical paradigm, with quantitative approach, its design is not of the experimental type applied and descriptive level, the population consisted of 137 households, the sample was 98 households, the sampling process was probabilistic (simple random sampling). The research techniques used were: the survey and direct observation. The results show a lack of physical infrastructure which is evident in the satisfaction of basic needs: education, health, housing, drinking water, basic sanitation and additionally a job with little chance of entering the labor market, albeit in possession of a high ecotourism potential represented by the presence of basins, pipes, streams and marshes, standing Basin Swamp Caimanera, with an area of 430 hectares. Inadequate and deficient public services infrastructure hampers the use of ecotourism as a potential development of the community.
\end{abstract}

Keywords: Ecotourism, communities, culture, infrastructure.

\section{Este artículo se puede referenciar}

Buelvas, C., Rojas, S. \& Tinoco, U (2015). Análisis socioeconómico y de potencialidades ecoturísticas en la ciénaga la Caimanera. En Desarrollo Gerencial Revista de la Facultad de Ciencias Económicas, Administrativas y Contables de la Universidad Simón Bolívar 7(1). 122-139.

\footnotetext{
${ }^{1}$ Proyecto de investigación: Ciénaga la Caimanera: un mundo de oportunidades para Coveñas a través del ecoturismo. Financiado por la Corporación Universitaria del Caribe (CECAR), del 13 de enero 2013 al 14 de mayo del 2014. Sincelejo (Colombia).

${ }^{2}$ Magíster en Administración de Empresas, docente de la Corporación Universitaria del Caribe, carlos.buelvas@cecar.edu.co.

${ }^{3}$ Magíster en Matemáticas Aplicada, docente de la Corporación Universitaria del Caribe, sandra.rojas@cecar.edu.co.

${ }^{4}$ Magíster en Administración de Empresas, docente de la Corporación Universitaria del Caribe, Ulises.tinoco@cecar.edu.co.
}

Desarrollo Gerencial, 7 (1) pp. 122 - 139 Enero-Junio 2015. ISSN: 2145-5147 (On Line). Universidad Simón Bolívar. 


\section{1.- INTRODUCCIÓN}

En el siglo XXI se consolida cierto turismo especializado, por el cual los demandantes tienen preferencia (Ministerio de Comercio Industria y Turismo, 2003) ${ }^{8}$. Este turismo es intensivo en el uso de mano de obra, característica que lo convierte en un factor dinamizador de la economía local en tanto contribuye a mejorar las condiciones de vida de las comunidades y la conservación del entorno (Organización Mundial del Turismo, 2003) ${ }^{9}$. Para prospectar esta tendencia en el municipio de Coveñas, es necesario conocer las condiciones de vida de la comunidad mediante la identificación de las variables socioeconómicas, culturales y de competencias de los habitantes de la zona de estudio, así como sus necesidades y potencialidades. Además es preciso identificar la infraestructura necesaria para la oferta de un turismo ecológico según Zamorano (2008).

Con este propósito se fijó como objetivo de la investigación realizar un análisis, con las variables más relevantes de tipo socioeconómico, culturales y de competencias, en el sector de la ciénaga la Caimanera, municipio de Coveñas; para su desarrollo se aplicó una encuesta a los residentes de la zona de estudio, que llevó a identificar cuáles son las potencialidades de las comunidades nativas y los aspectos socioculturales que podrían constituirse en un fuerte atractivo del ecoturismo en la zona. Dicha información permitió la respectiva caracterización (edad, género, estado civil, nivel de ingreso, nivel educativo, fuente de trabajo, condiciones de la vivienda, etc.), y de igual manera se determinó tanto la demanda de este tipo de turismo como la infraestructura existente para cubrirla.

El análisis socioeconómico presentó con claridad la escasa inversión estatal ante la manifiesta deficiencia en los servicios públicos básicos, y en los sectores de salud y vivienda, lo que dificulta el desarrollo de las actividades ecoturísticas, a pesar de la enorme riqueza potencial de los recursos naturales y culturales, en especial las cuencas, caños, arroyos y ciénagas que interactúan con el mar creando condiciones específicas para el desarrollo del bosque de manglar, flora y fauna asociada.

Dentro de la fundamentación teórica que respaldó la investigación se consideró el concepto de desarrollo sostenible ante la necesidad de hacer un uso cada vez más racional de los recursos existentes en el planeta, y de contribuir de manera más equitativa la riqueza social, actuando conjuntamente para evitar que la acción del hombre deteriore el medio ambiente (hasta niveles irreversibles), o comprometa la existencia de las generaciones futuras (Díaz, 2009). Dicha teoría se complementa con los axiomas del ecoturismo acerca de minimizar los impactos negativos que la actividad genera para el ambiente y para la comunidad, y de construir respeto y conciencia ambiental y cultural. Esto induce a una práctica

${ }_{9}^{8}$ MCTI (Ministerio de Comercio Turismo e Industria).

${ }^{9}$ OMT (Organización Mundial del Turismo).

Desarrollo Gerencial, 7 (1) pp. 122 - 139 Enero-Junio 2015. ISSN: 2145-5147 (On Line). Universidad Simón Bolívar. 


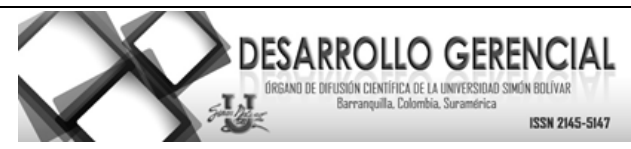

equilibrada del ecoturismo entre la conservación y el desarrollo a través de la promoción de las relaciones sinérgicas en las áreas naturales conforme señalan Ross (1999) y Vanegas (2006).

Ante los atractivos naturales de la ciénaga la Caimanera, las potencialidades socio-culturales de la comunidad receptora y la necesidad de disfrutar unas vacaciones placenteras sin causar daño a la naturaleza, se formuló la siguiente pregunta: ¿Cuáles son las condiciones socioeconómicas y las potencialidades ecoturísticas en la ciénaga la Caimanera, del municipio de Coveñas?

\section{2.- MÉTODO}

\section{Diseño}

La investigación se basó en un diseño no experimental cuantitativo de tipo aplicado y nivel descriptivo; fue realizada bajo la óptica del paradigma empírico-analítico, y se desarrollo entre los años 2012-2014, planeándose en cuatro etapas. En la primera se planificó el trabajo, acopió y clasificó la bibliografía; en la segunda se practicaron las visitas de campo y se aplicaron las técnicas que se habían definido en el estudio; en la tercera se llevó a cabo la tabulación y análisis de los datos recogidos y, por último, la socialización de los resultados de la investigación con la comunidad correspondió a la cuarta etapa. El procesamiento y análisis de la información se efectuó mediante el uso herramientas ofimáticas tales como Excel y Project.

El criterio de inclusión tenido en cuenta en la investigación fue la permanencia y residencia de las personas que conforman el hogar; y el criterio de exclusión se determinó por la no permanencia en la zona. El tamaño de la muestra determinado fue de 98 hogares de la ciénaga la Caimanera; el análisis de la información se realizó empleando técnicas y medidas estadísticas de tipo descriptivo; además, se realizaron tablas de contingencia para hacer análisis bivariado (ver gráfica 2).

\section{Participantes}

La población objeto de estudio está conformada por $N=137$ hogares de la ciénaga la Caimanera, determinados por conteo físico a partir de la observación directa. El tamaño de la muestra se calculó mediante la fórmula estadística (ver fórmula 1) para una población finita y conocida, con un nivel de confianza del $95 \%$. Ello implica que $Z_{c}=1.96$. Donde $p$ es la probabilidad de que un hogar sea seleccionado tomándose $p=0.50, \mathrm{y}(1-p)$ es la probabilidad de que un hogar no sea seleccionado siendo $(1-p)=0.50$ y un error $E=5 \%$. Se obtiene una muestra $n=98$ hogares. 


$$
n=\frac{Z_{C}^{2} \times p(1-p) \times N}{E^{2} \times(N-1)+Z_{C}^{2} \times p(1-p)}
$$

Empleando el método aleatorio simple, el proceso de muestreo fue probabilístico; así, todos los hogares de la población ciénaga la Caimanera tenían igual susceptibilidad de ser seleccionados. Para la técnica de observación directa se consideraron los trabajos de Ospina \& Mora (2013), Romero (2008) y De Almeida (2011).

\section{Instrumentos}

Las técnicas de investigación utilizadas fueron: la encuesta y la observación directa. El instrumento aplicado fue un cuestionario empleado en la gran encuesta integral de hogares del DANE (2005), que se complementó con ítems relacionados con las variables culturales y de competencias ecoturísticas; dicho cuestionario fue aplicado a los miembros cabeza de hogar residente. La principal motivación para aplicarlo es su validación respaldada por el DANE (2005); sin embargo, considerando la adición de nuevos ítems se calculó el coeficiente alfa de Cronbach con un valor del 94\%, lo cual describe su validez y nivel de confiabilidad.

Los instrumentos empleados en la observación directa fueron el diario y registros anecdóticos. Se realizaron varias visitas a la zona de estudio, se elaboró un formato para la toma de apuntes, y cuando no era posible, se tomaron las conversaciones con las personas residentes como registro de evidencia en cada lugar de interés para la investigación, utilizando cámaras fotográficas o de video. Estos registros descriptivos permitieron determinar en la unidad muestral (hogares) tanto las características de inclusión como las de las viviendas y el inventario destinado a las prácticas del ecoturismo. La información recopilada llevó al logro de los objetivos de esta investigación.

\section{Procedimientos}

En un primer momento se acopió y clasificó la documentación pertinente con el fin de extraer la información que sirvió de base para conformar la fundamentación teórica. Posteriormente, se realizó el diseño metodológico y la construcción de los instrumentos de recolección de la información: encuesta y el instrumento de la observación directas; después se realizaron visitas a la comunidad para socializarle el proyecto y motivar la aceptación de los investigadores; luego se aplicó una prueba piloto y se realizó la validación de la encuesta, que permitió la reestructuración del instrumento para su aplicación definitiva. Esta fue realizada por personas de la misma comunidad a fin de posibilitar la veracidad de la información. 


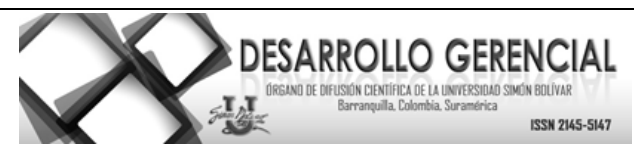

Durante las visitas de campo simultáneamente se aplicó la observación directa en el reconocimiento de infraestructura física que pudiera destinarse al ecoturismo.

\section{3.- RESULTADOS}

\section{Variables socioeconómicas}

\section{a) Educación}

Los resultados obtenidos muestran que el $45 \%$ de esta población es bachiller y el $26 \%$ tiene formación básica primaria; la educación técnica, tecnológica y profesional es del 13\%, 1\% y 6\%, respectivamente (ver gráfica 1), lo cual difiere sustancialmente de la información sobre el acceso a la educación superior en el municipio de Coveñas. Otro aspecto que incide en la formación son las instalaciones locativas, pues de acuerdo a los estándares de calidad son inadecuadas; la dotación de salas de informática es escasa y en la mayoría de los casos es inexistente, y los pocos equipos del área disponible son obsoletos, por lo cual no permiten la articulación con las entidades de educación superior y limitan las posibilidades de acceder a nuevos ambientes de aprendizaje (Plan de Desarrollo Municipal, 2012).

\section{Gráfica 1: Distribución población en edad de trabajar según nivel de formación PET $^{10}$}

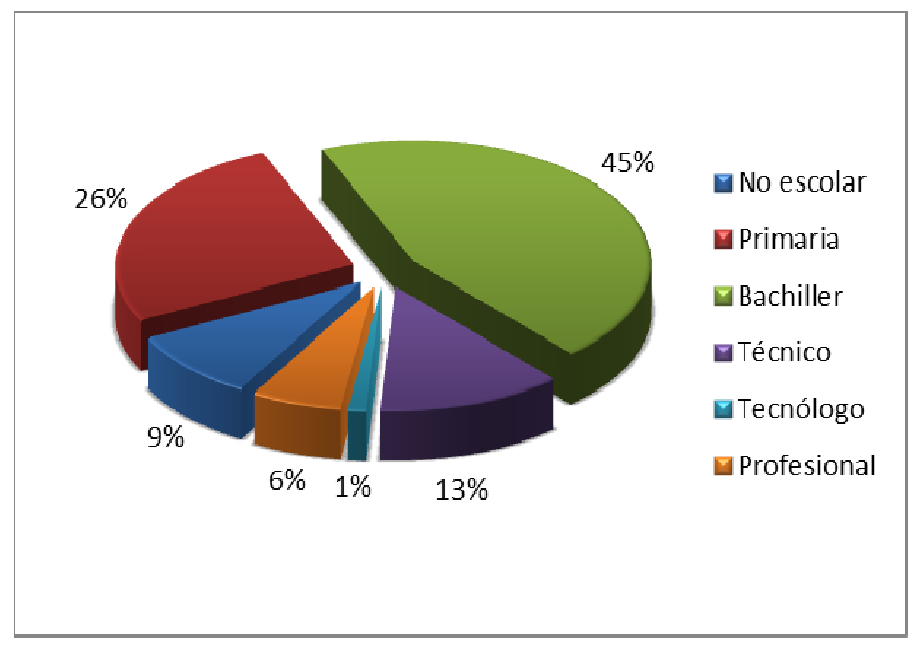

Fuente: grupo investigador.

\section{b) Salud}

La cobertura en salud de los hogares en la ciénaga la Caimanera es del 100\% dentro del régimen subsidiado; sin embargo, la calidad de la prestación del servicio no es buena, y se debe recibir en el Centro

${ }^{10}$ PET (Población en edad de trabajar). 
de Salud de Coveñas, "el cual no cuenta con los equipos ni la infraestructura física adecuada y en épocas de invierno se inunda" (Plan de Desarrollo Municipal, 2012).

\section{c) Infraestructura y medio ambiente}

En la ciénaga la Caimanera la situación es precaria; la cobertura en la prestación de acueducto y alcantarillado atiende el $2 \%$ de las viviendas, lo que constituye una limitante para el desarrollo sostenible de la actividad turística en esta zona (DNP, 2010) ${ }^{11}$. Esta realidad lleva a que el 91\% de las viviendas, se abastezca de agua proveniente de pozos profundos, pluviales o de carrotanques, sin tratamiento de potabilización, con la consiguiente exposición (en materia de enfermedades gastrointestinales) a la población nativa y turistas; adicionalmente, el $87 \%$ de las viviendas posee pozos sépticos mientras las aguas servidas se viertan a las calles y arroyos que tributan al ecosistema de la ciénaga la Caimanera, a lo que se suma el deficiente servicio de aseo (35\%). En cuanto al servicio de energía eléctrica, el 100\% de los hogares lo recibe; el 45.9\% cuenta con el servicio de gas natural; el 3.06\% tiene teléfono fijo y el $12.24 \%$ dispone de internet (ver gráfica 2).

\section{Gráfica 2: Servicios públicos Ciénaga la Caimanera, 2014.}

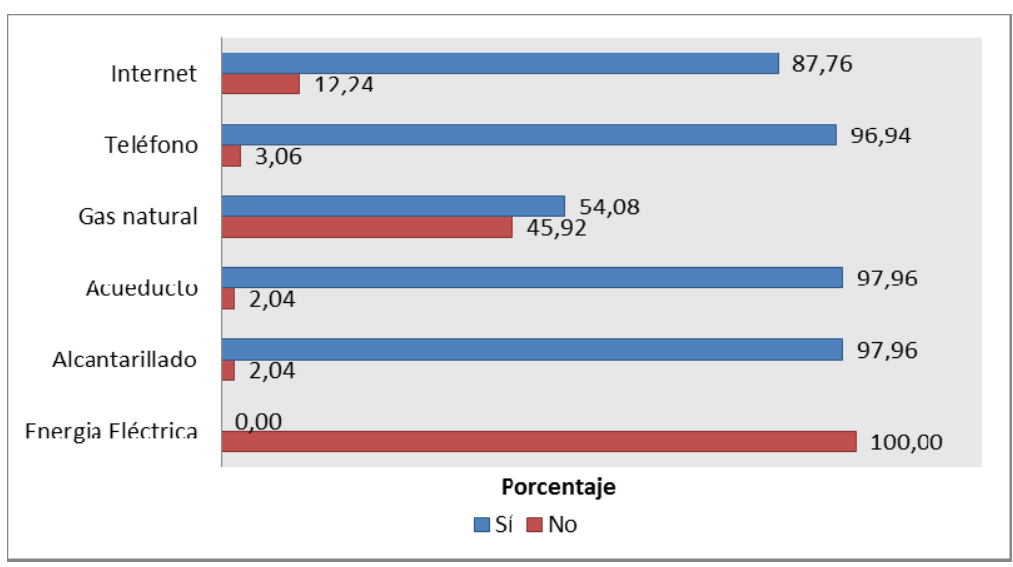

Fuente: grupo investigador

\section{d) Vivienda}

El estudio evidenció que el 92\% de las viviendas fueron diseñadas como casas de habitación y el 5\% tipo apartamento. Además, se encontró que el $63 \%$ de quienes residen en las viviendas son propietarios

${ }^{3}$ PET (población en edad para trabajar).

11 DNP (Dirección Nacional de Planeación). 


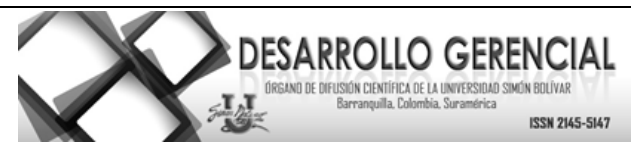

mientras el 29\% de las familias tienen el bien en usufructo y el 6\% vive en arriendo o subarriendo. En cuanto al usufructo en la Ciénaga la Caimanera, su alta proporción se explica por la labor que desempeñan los jefes del hogar: residen y laboran como empleados domésticos y vigilantes de cabañas y casas de recreo. El material de construcción de los muros de las viviendas es de ladrillo en un $70 \%$, madera burda en un $22 \%$, bahareque un $5 \%$ y guadua $3 \%$; en lo referente a los pisos, el $46 \%$ es material de cemento o gravilla; el $32 \%$, baldosas o tabletas y el $20 \%$ solo es de tierra apisonada (Fuente: grupo investigador).

Adicionalmente, en esta comunidad se encontró que existe un hacinamiento equivalente al 51.02\% (ver gráfica 3). Tomando como referente la definición hacinamiento dada por el DANE (2011) "existe hacinamiento cuando hay en promedio tres o más personas del hogar por cada cuarto", la muestra determinada en la zona de la Ciénaga la Caimanera está conformada por 98 hogares, para un total de 422 personas. Y se encontró que 50 de los 98 hogares en cuestión están hacinados.

Gráfica 3: Número de personas por grupo familiar vs. Número de cuartos por grupo familiar 2014

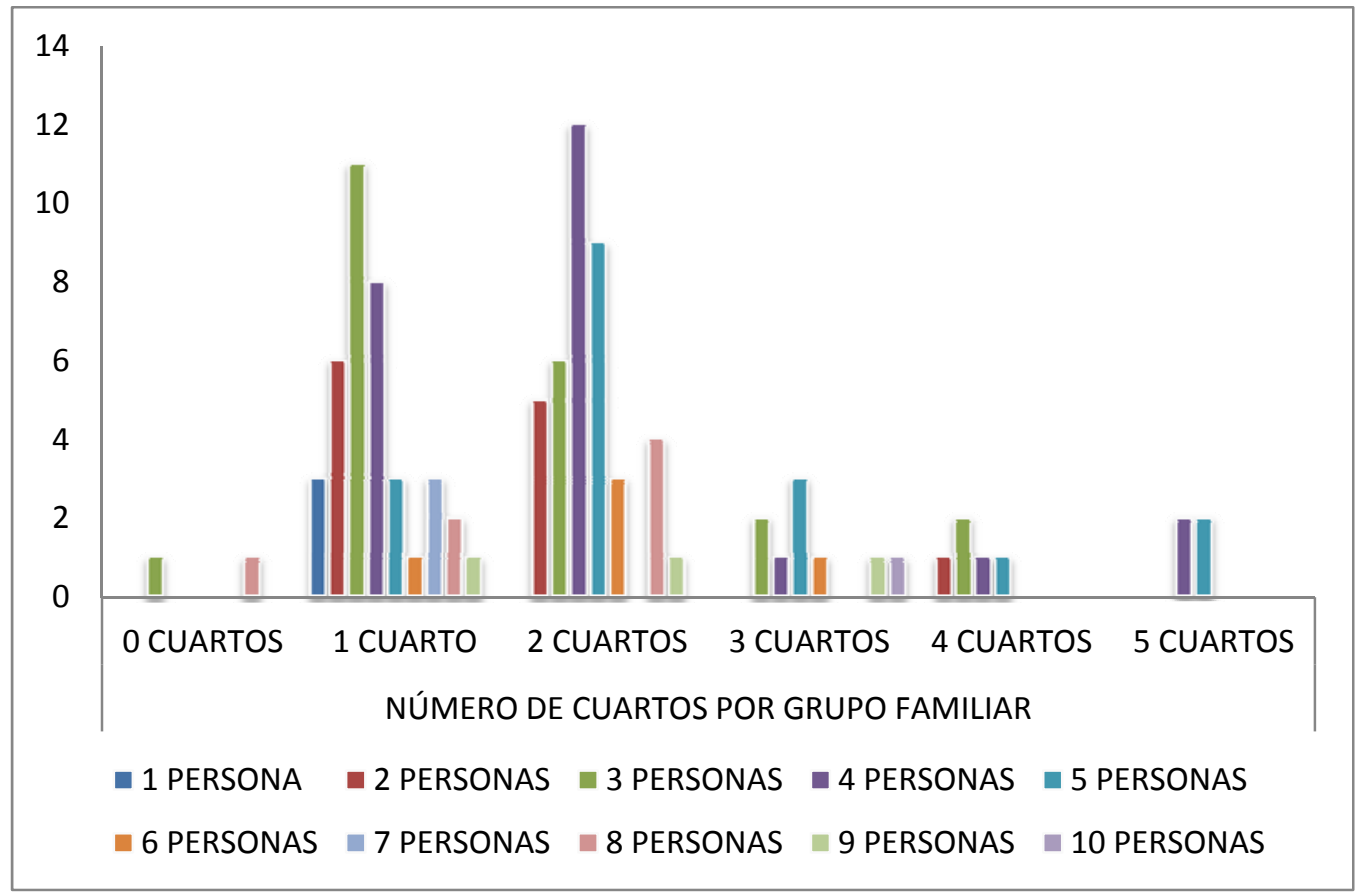

Fuente: grupo investigador.

\section{e) Demografía}

La dinámica poblacional, así como su estructura quedó reflejada en los resultados de la encuesta practicada, en donde se muestra que el $47,87 \%$ son representantes del género masculino y el 52,13\%, del género femenino; la proporción de mujeres en la zona objeto de estudio supera a la población de 


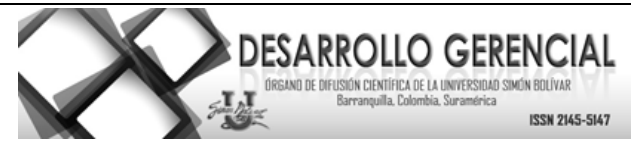

hombres; en cuanto al índice de masculinidad, ${ }^{12}$ en el municipio de Coveñas, es de 103.8; en el departamento de Sucre es de 102.76 y a nivel nacional es de 97.5 hombres por cada 100 mujeres (DANE, 2005). Lo anterior contrasta con el dato de la ciénaga la Caimanera, que es de 91.8 hombres por 100 mujeres (tabla 1).

Tabla 1. Distribución de la población por edad y género en la ciénaga la Caimanera, 2014

\begin{tabular}{|c|c|c|c|c|c|c|}
\hline $\begin{array}{c}\text { GRUPOS DE } \\
\text { EDADES }\end{array}$ & $\begin{array}{c}\text { POBLACIÓN } \\
\text { TOTAL }\end{array}$ & HOMBRES & $\begin{array}{c}\text { \% DEL } \\
\text { TOTAL(H) }\end{array}$ & MUJERES & $\begin{array}{c}\text { \% DEL } \\
\text { TOTAL(M) }\end{array}$ & $\begin{array}{c}\text { \% } \\
\text { TOTAL }\end{array}$ \\
\hline $0-4$ & 39 & 16 & $3,79 \%$ & 23 & $5,45 \%$ & $9 \%$ \\
\hline $5-9$ & 41 & 23 & $5,45 \%$ & 18 & $4,27 \%$ & $10 \%$ \\
\hline $10-14$ & 47 & 27 & $6,40 \%$ & 20 & $4,74 \%$ & $11 \%$ \\
\hline $15-19$ & 38 & 22 & $5,21 \%$ & 16 & $3,79 \%$ & $9 \%$ \\
\hline $20-24$ & 54 & 18 & $4,27 \%$ & 36 & $8,53 \%$ & $13 \%$ \\
\hline $25-29$ & 45 & 22 & $5,21 \%$ & 23 & $5,45 \%$ & $11 \%$ \\
\hline $30-34$ & 27 & 12 & $2,84 \%$ & 15 & $3,55 \%$ & $6 \%$ \\
\hline $35-39$ & 18 & 7 & $1,66 \%$ & 11 & $2,61 \%$ & $4 \%$ \\
\hline $40-44$ & 29 & 12 & $2,84 \%$ & 17 & $4,03 \%$ & $7 \%$ \\
\hline $45-49$ & 32 & 19 & $4,50 \%$ & 13 & $3,08 \%$ & $8 \%$ \\
\hline $50-54$ & 19 & 7 & $1,66 \%$ & 12 & $2,84 \%$ & $5 \%$ \\
\hline $55-59$ & 11 & 5 & $1,18 \%$ & 6 & $1,42 \%$ & $3 \%$ \\
\hline $60-64$ & 10 & 8 & $1,90 \%$ & 2 & $0,47 \%$ & $2 \%$ \\
\hline $65-69$ & 2 & 0 & $0,00 \%$ & 2 & $0,47 \%$ & $0 \%$ \\
\hline $70-75$ & 1 & 1 & $0,24 \%$ & 0 & $0,00 \%$ & $0 \%$ \\
\hline $75-79$ & 3 & 0 & $0,00 \%$ & 3 & $0,71 \%$ & $1 \%$ \\
\hline $80-84$ & 4 & 2 & $0,47 \%$ & 2 & $0,47 \%$ & $1 \%$ \\
\hline+85 & 2 & 1 & $0,24 \%$ & 1 & $0,24 \%$ & $0 \%$ \\
\hline & 422 & 202 & 0,4787 & 220 & $52,13 \%$ & $100 \%$ \\
\hline
\end{tabular}

Fuente: grupo investigador.

\section{f) Distribución de la población por edad y género}

La estructura poblacional en la ciénaga la Caimanera muestra que su base está conformada fundamentalmente por la población en edad inactiva ${ }^{13}$, (ver tabla 1) en una proporción aproximada del $30 \%$. Adicionalmente, la razón de niños por mujeres (RNM) ${ }^{14}$ en la ciénaga la Caimanera es del 29.8\%, y

\footnotetext{
12 Índice de masculinidad se calcula dividiendo la población total de hombres entre la población total de mujeres, que indica la cantidad de hombres por cada 100 mujeres.

${ }^{13}$ Edad inactiva: Constituida por niños, niñas y adolescentes que se encuentran por lo general en proceso de educación preescolar y básica primaria y con una alta dependencia económica de sus hogares.

${ }^{14}$ RNM: Según el DANE se calcula dividiendo la población menor de 5 años entre las mujeres en edad fértil (15 a 49 años).
} 


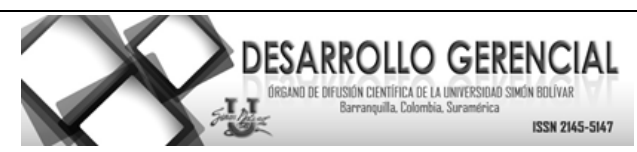

en lo referente a la población con edades de 60 y más años; en la ciénaga la Caimanera es de 5,21\%. Se observa que la población es joven en su mayoría (ver grafica 4).

\section{Gráfica 4: Pirámide poblacional Ciénaga la Caimanera, 2014}

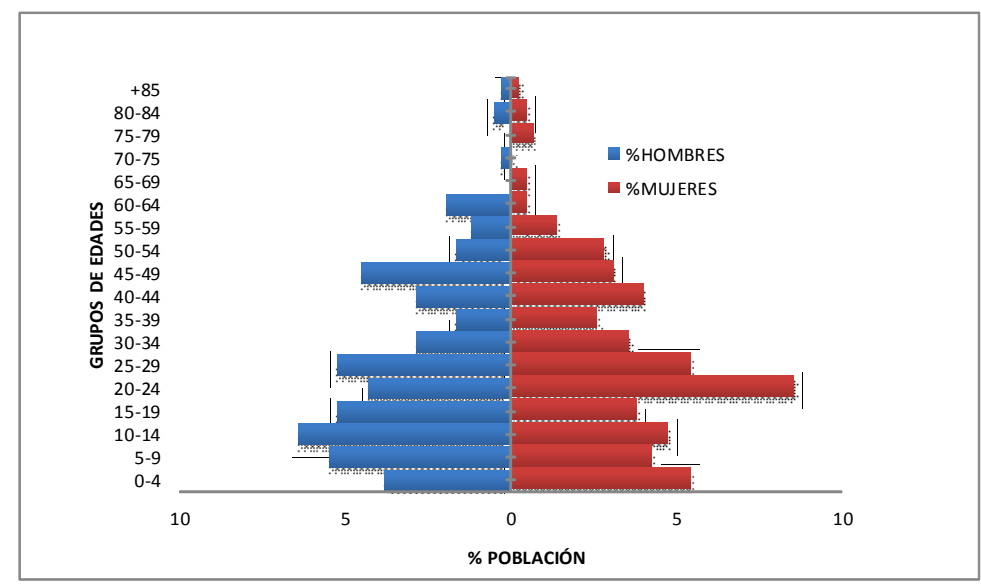

Fuente: grupo investigador.

\section{g) Pertenencia étnica}

En la ciénaga la Caimanera las personas residentes tienen un fuerte arraigo con sus ancestros: el 43\% se autorreconoce como negro, mulato o raizal, y el 5\% como indígena.

Gráfica 5: Porcentaje de pertenencia étnica, ciénaga la Caimanera, 2014

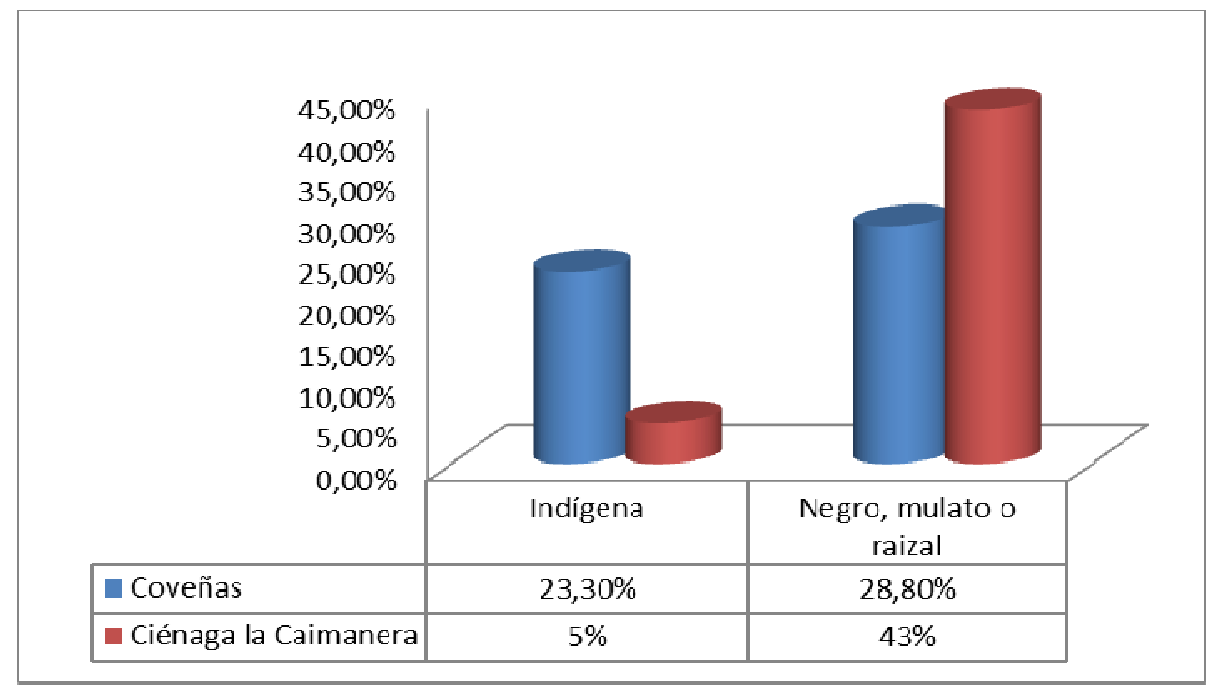

Fuente: grupo investigador. 


\section{Aspectos socioculturales}

\section{a) Organizaciones comunitarias}

El $60 \%$ de la población en la ciénaga la Caimanera se encuentra actualmente vinculada a alguna organización comunitaria, pero el 36\% no lo está. Al indagar el tipo de agremiación a la que pertenece la población, el $71 \%$ está afiliado a una organización de base; el 11\% está asociada a cooperativa; el 43\%, a la acción comunal y 17\%, a otras organizaciones. El 29\% de la población no responde (ver gráfica 6).

\section{Gráfica 6: Tipo de agremiación Ciénaga la Caimanera 2014}

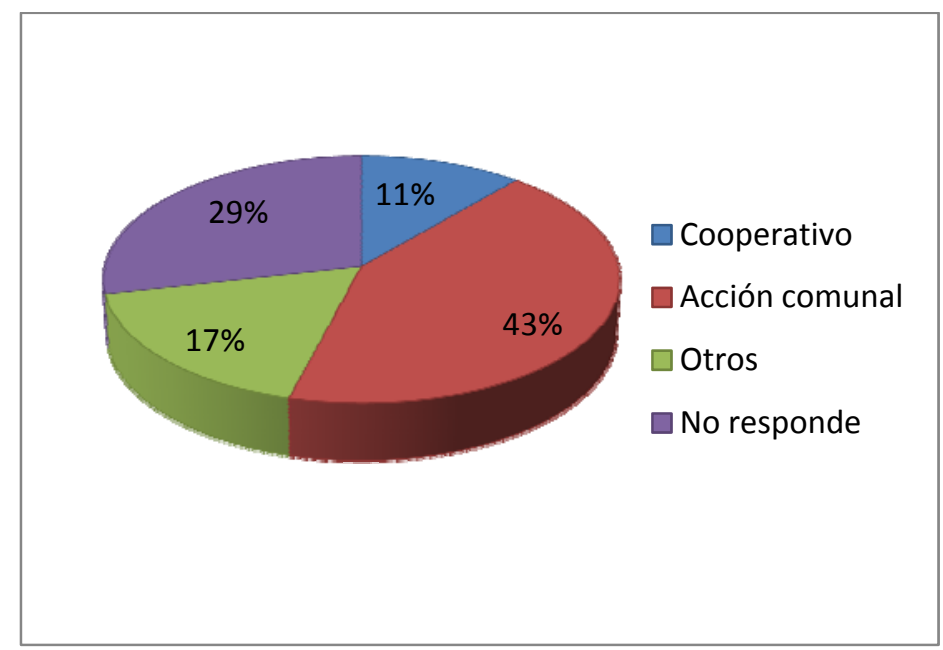

Fuente: grupo investigador.

\section{b) Generación de ingresos}

En un 53\% de los hogares encuestados se realizan actividades económicas en la misma vivienda especialmente venta de alimentos, licores y bebidas; no obstante, en materia de ingresos, para el 76\% de los hogares lo percibido no alcanza a cubrir los gastos familiares; tal porcentaje revela una condición muy crítica cuando se compara a nivel nacional y regional, en donde apenas resulta ser del 32,7\% y 39\%, respectivamente (ver gráfica 8). Sobre las opciones para aumentar los ingresos, el 57\% afirmó que la educación es la mejor; el 24\% señala el empleo como solución y el 12\% ve la alternativa en el ecoturismo. El menor porcentaje corresponde al comercio y la agricultura.

Se destaca que en el $65 \%$ de los hogares recibieron capacitación relacionada con la actividad turística que ha brindado el SENA regional Sucre; esto ha permitido que el $79 \%$ de la población se oriente a laborar en calidad de guía turístico, un $7 \%$ al transporte de turistas y el $14 \%$ restante desarrolla actividades de apoyo y conexas al turismo, como son: los servicios de bar, restaurante, culinaria, aseo, 


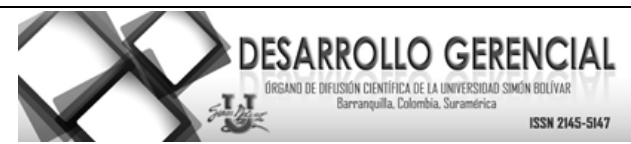

vigilancia de parqueaderos, ventas de artesanías, entre otros. Ello contrasta con la información aportada (ver gráfica 7).

\section{Gráfica 7: Oferta de servicios en ciénaga la Caimanera}

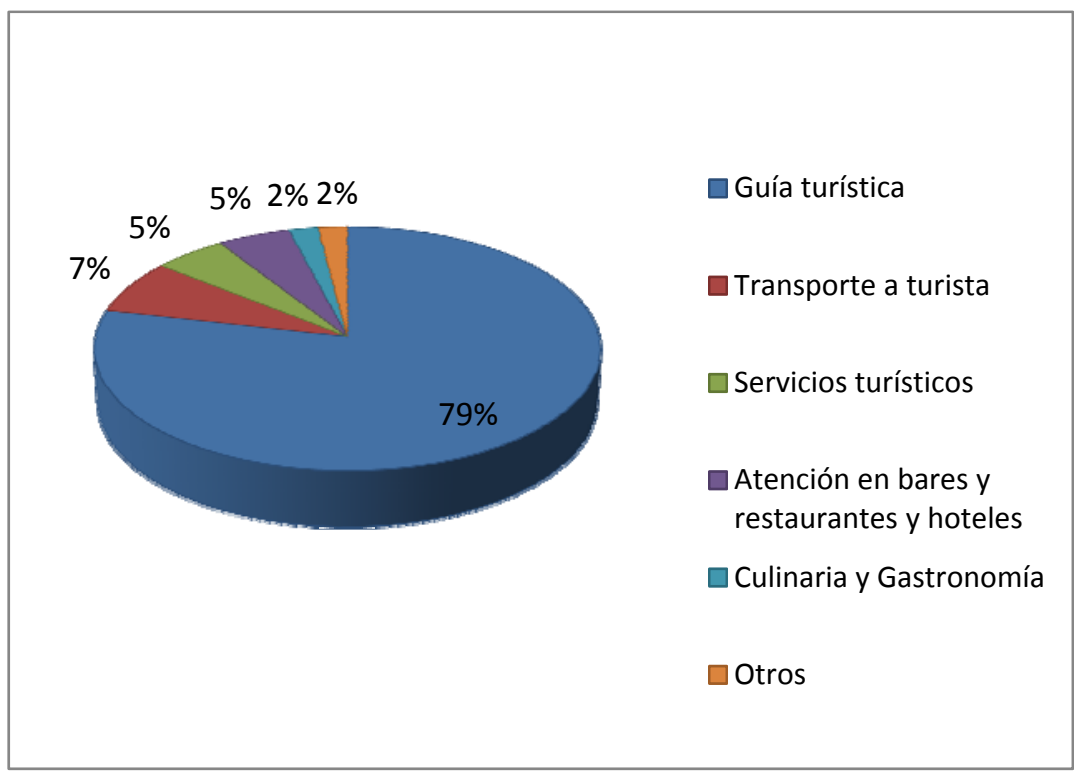

Fuente: grupo investigador.

Gráfica 8: Porcentaje de hogares con suficiencia o insuficiencia de ingresos: ciénaga la Caimanera, Costa Atlántica y Nacional

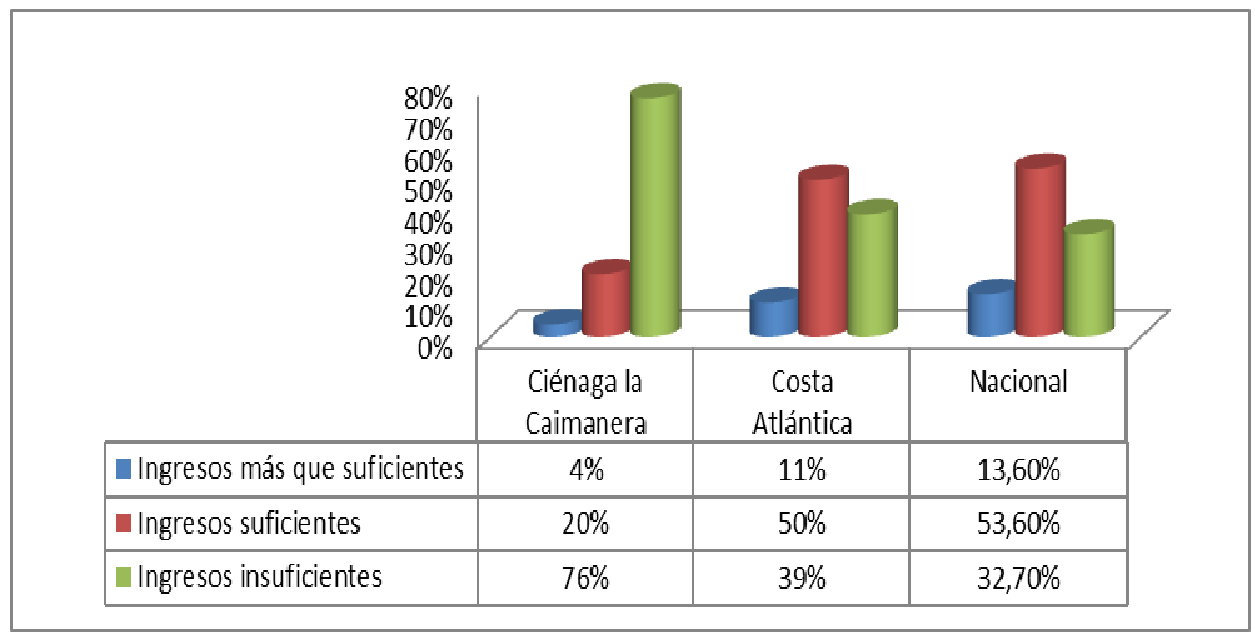

Fuente: grupo investigador.

Adicionalmente, el 57\% identifica la importancia que tiene el ecoturismo en la zona, incluso reconoce el potencial del contexto en la generación de ingresos. Al respecto preocupa que los peces, la babilla y el caimán son los animales con mayor tendencia hacia la extinción. (Fuente: grupo investigador). 
Actual infraestructura destinada a la prestación de los servicios de ecoturismo

A través de la observación directa, el inventario realizado permitió determinar la infraestructura ecoturísticas existente en la ciénaga la Caimanera como elemento para la dinamización de los atractivos naturales y culturales. El inventario constituye un registro y un estado integrado de todos los elementos turísticos que por sus cualidades naturales, culturales y humanas pueden constituir un recurso para el turista; de esta manera representa un instrumento valioso para la planificación turística, toda vez que sirve como punto de partida al realizar evaluaciones y establecer las prioridades necesarias para el desarrollo turístico nacional (mincetur.gob.pe, 2006).

Para la elaboración de este inventario se procedió a la recolección, análisis y clasificación de la infraestructura existente con miras a desarrollarla como atractivo ecoturístico. De las visitas de campo realizadas, diálogos con los guías turísticos y la percepción de los habitantes del sector se determinó que la infraestructura existente dedicada al ecoturismo está constituida por:

- Casa flotante al interior de la ciénaga ${ }^{15}$

- Senderos fluviales al interior de los manglares.

- Acuario Golfo Verde (iniciativa privada).

- Medios de transporte fluvial (canoas).

\section{4.- DISCUSIÓN}

El objetivo de la investigación consistió en realizar un análisis socioeconómico y de potencialidad ecoturísticas como medio de desarrollo de la comunidad de la ciénaga la Caimanera, con base en la biodiversidad (fauna y flora), la variedad paisajística propia de los sitios de manglar, sabanas, playas y la etnografía asociada a la cultura manifestada en variedad gastronómica y festividades tradicionales. Evidenciando que esta zona cuenta con recursos naturales que de ser tratados eficientemente pueden convertirse en el medio para que esta comunidad se desarrolle, [...] el «crecimiento» es el medio y el «desarrollo» es el fin”, Silva (2010). Desde este enfoque Ochoa (2013) considera el ecoturismo como una oportunidad para el desarrollo, la conservación y la generación de beneficios a las comunidades. Es asi como la organización Mundial del turismo define siete formas en las que los pobres pueden beneficiarse del turismo. (Van der Duim, 2007).

Los resultados de la investigación arrojaron que la población tiene una tasa de analfabetismo del 9\%, menor que la del municipio de Coveñas, que es del 18\% (Plan de Desarrollo Municipal, 2012), Sin

${ }^{15}$ Al momento de redactar este artículo se conoció que la casa flotante sufrió un accidente y quedó fuera de servicio luego de un incendio.

Desarrollo Gerencial, 7 (1) pp. 122 - 139 Enero-Junio 2015. ISSN: 2145-5147 (On Line). Universidad Simón Bolívar. 


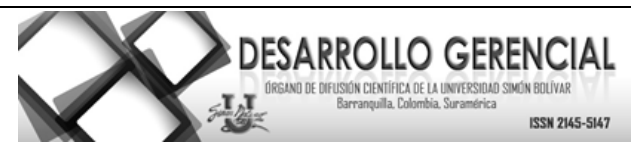

embargo, el acceso a la educación superior tiene mayor cobertura en el municipio de Coveñas, que presenta mayor demanda en los Ceres ${ }^{16}$ (Plan de Desarrollo Municipal, 2012). La baja participación de la población de la ciénaga la Caimanera en la educación superior, se convierte en una limitante para desarrollar sus capacidades y potencialidades, según lo define Sen (1999). En ese sentido, la Cepal ${ }^{17}$ (2000) establece que "la escasez de capital humano influye en el acceso a determinados empleos, y la carencia de conocimientos técnicos o tecnológicos restringe la posibilidad de generar y desarrollar una iniciativa empresarial". Por lo tanto, "la ausencia de capital educativo predispone a los individuos [...] y los condiciona fuertemente a que en un futuro sean seguros candidatos a diversas formas de pobreza y/o exclusión” (Naranjo, 2002).

Consecuentemente, la cobertura en salud de los hogares en la ciénaga la Caimanera se da totalmente (100\%) en el régimen subsidiado, es decir, que supera a la del municipio de Coveñas que es del 93\% (Plan de Desarrollo Municipal, 2012); además, sobrepasa el promedio de la Costa Atlántica y la media nacional, 60,2\% y 49,7\%, respectivamente (DANE, 2014). Esto significa que se trata de una población sumida en la pobreza y con alta dependencia de los programas sociales del Estado, con el agravante, de unos servicios deficientes en el Centro de Salud de Coveñas por causa de su inadecuada infraestructura física, que en épocas de invierno también se inunda (Plan de Desarrollo Municipal, 2012). Lo anterior repercute negativamente en la capacidad laboral, en el estado de la salud física y mental, la esperanza de vida y en las tasas de mortalidad de las personas (Álvarez, 2009).

Por otro lado, en el municipio de Coveñas la cobertura del acueducto es del 82,3\% y la del alcantarillado, 70,55\% (Plan de Desarrollo Municipal, 2012); en el ámbito nacional, la cobertura del acueducto es del 87.3\% y la del alcantarillado, 72.3\% (DANE, 2014). Mientras tanto en la ciénaga la Caimanera, la cobertura en materia de servicios domiciliarios de acueducto y alcantarillado es del 2\%; si se comparan estos datos con la media nacional, el servicio de energía eléctrica es similar (97.4\%), pero los otros restantes están por debajo del promedio nacional, y “[...] se constituye en un limitante para el desarrollo sostenible de la actividad turística en esta zona" (DNP, 2011).

A su vez, los jefes de hogar de la ciénaga la Caimanera realizan actividades económicas en sus viviendas y de prestación de servicios turísticos, y son conscientes de que la educación es la mejor opción para mejorar sus ingresos: el $12 \%$ asume el ecoturismo como una fuente para generar ingresos, de hecho se consideren competentes en trabajos que tienen relación directa con el ecoturismo. Se destaca que el $65 \%$ de los hogares han recibido capacitación relacionada con esta actividad brindada por el SENA regional Sucre; sin embargo, un alto porcentaje manifiesta que sus ingresos son insuficientes.

${ }^{16}$ Centros regionales de educación superior.

${ }^{17}$ CEPAL (Comisión Económica para América Latina). 


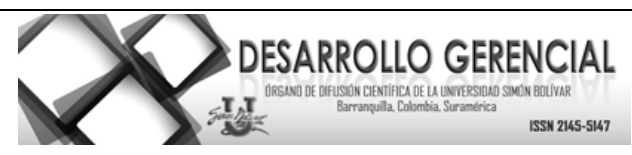

Lo anterior implica una forzosa emigración, generalmente relacionada "con la falta de trabajo, la inseguridad como producto de la violencia, los problemas socioeconómicos, el mejoramiento de la calidad de vida, la búsqueda de desarrollo individual o familiar, oportunidades y educación, acceso a bienes y servicios, entre otras" (Aruj, 2008). En la ciénaga la Caimanera los factores que inciden en la emigración del nativo son: la falta de oportunidades laborales, así como la búsqueda de un mejoramiento de la calidad de vida y oportunidades de educación. (Fuente: grupo investigador.)

Por tanto, es de gran importancia la capacitación para la prestación de los servicios ecoturístico dado el tipo de información que se maneja y las competencias que se desarrollan: "la capacitación para el servicio ecoturístico se orienta a todas las personas que directa o indirectamente laboren en actividades ecoturísticas, asegurando que ésta, además de manejar contenidos de alto valor científico, incorporará la sabiduría tradicional de los pobladores de los lugares [en] donde [estas] se desarrollan [...]" (Méndez, 2008). En este sentido, Prada (2009) expresa: "este turismo alternativo ambiental da inicio al ecoturismo, centrado en la necesidad de conservar o preservar la naturaleza, además de generar ingresos que satisfagan las necesidades de la comunidad".

Con base en la proyección de la población del DANE hasta el año 2009, se destaca que la población del municipio de Coveñas es joven, dado que el $53.72 \%$ tiene edades menores a 25 años mientras la población de la ciénaga la Caimanera tiene una proporción similar: 52\% (tabla 1). Esto reviste especial importancia en la medida en que estos grupos etarios están en proceso de formación, y constituye un reto para el sistema educativo y para estimular la demanda de empleo dirigido a dicho segmento poblacional. Como la proporción de mujeres es mayor que la de los hombres (ver gráfica 4), el género se convierte en una variable demográfica importante, puesto que las conductas y los roles están asociados al comportamiento de las personas con relación a la fecundidad, la mortalidad y las migraciones (Schkolnik, 2010).

El ecoturismo busca la recreación, el esparcimiento y la educación del visitante a través de la observación, el estudio de los valores naturales y de los aspectos culturales relacionados con ellos, tal como lo contemplan la ley 300 (1996) y la Ley 1558/2012 ${ }^{18}$; en este sentido, el hecho de que el $43 \%$ de los jefes cabeza de hogar se autorreconocen como negro, mulato o raizal y el 5\% como indígena permite la conservación de tradiciones y, por ende, el intercambio cultural con los turistas.

En cuanto a los resultados referidos a la vivienda, se evidenció que para su construcción se utiliza principalmente el ladrillo en un $70 \%$ y madera burda en un $22 \%$, los pisos en un $46 \%$ son de cemento o gravilla; el $32 \%$, baldosas o tabletas y el $20 \%$ es de tierra apisonada; además se encontró hacinamiento del

${ }^{18}$ Ley 1558/2012: Ley general de turismo.

Desarrollo Gerencial, 7 (1) pp. 122 - 139 Enero-Junio 2015. ISSN: 2145-5147 (On Line). Universidad Simón Bolívar. 


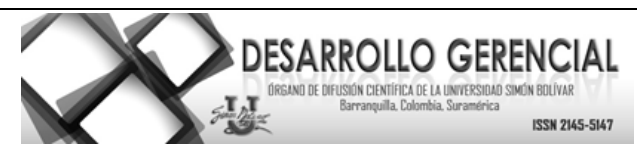

$52,02 \%$ lo que en conjunto refleja condiciones habitacionales inadecuadas. El resultado contrasta con el concepto de desarrollo humano sostenible concebido por MCTI (2003), que por su enfoque integral pasa por el análisis de muchas variables, como son: eliminación de la pobreza, reducción del crecimiento demográfico, una distribución más equitativa de los recursos, todo lo cual va en contradicción de las condiciones habitacionales de la ciénaga la Caimanera.

En la ciénaga la Caimanera se encuentran fuertes vínculos a través de los procesos de organización comunitaria. El $60 \%$ de los jefes de hogar pertenecen a una organización comunitaria, condición que facilitaría la práctica del ecoturismo en la zona en la medida en que actualmente el turismo implica la relación de múltiples sectores de la sociedad: empresarios, entidades del gobierno, en el origen, comunidades receptoras, instituciones educativas, organizaciones no gubernamentales en el destino, tal y como lo plantea $\mathrm{OMT}^{19}$.

\section{CONCLUSIONES}

La comunidad de la ciénaga la Caimanera presenta altas deficiencias en la satisfacción de sus necesidades básicas, especialmente lo relacionado con los servicios de acueducto, aseo y alcantarillado, que prácticamente constituyen las necesidades más sentidas, puesto que conllevan bajos niveles de las condiciones de vida de la población, con impactos negativos en el medio ambiente y los recursos naturales de la zona. Así mismo es un serio limitante para dinamizar las actividades económicas orientadas a la generación de empleo e ingresos relacionados con el ecoturismo, no obstante los sitios ecológicos y riquezas naturales de gran atracción que posee el lugar.

En este marco, y más por un proceso de adaptación empírica, y no tanto como un proceso de planificación consciente de la actividad, la población de la ciénaga la Caimanera ha venido desarrollando una economía de subsistencia e insertándose precariamente en algunas actividades empresariales conexas con el ecoturismo, es decir, de labores temporales y por cuenta propia, que a la postre le permite obtener ingresos si bien resultan ser insuficientes para el sostenimiento de los gastos básicos de los hogares.

Lo anterior permite plantear una mayor intervención del municipio de Coveñas mediante políticas públicas orientadas especialmente a la ampliación de la cobertura de los servicios de acueducto, aseo y alcantarillado, y que permitiría a los hogares estar en condiciones de mejorar su calidad de vida, pero también de brindar adecuados servicios a los turistas y diversificar sus actividades productivas de apoyo al ecoturismo.

19 OMT (Organización Mundial de Turismo).

Desarrollo Gerencial, 7 (1) pp. 122 - 139 Enero-Junio 2015. ISSN: 2145-5147 (On Line). Universidad Simón Bolívar. 


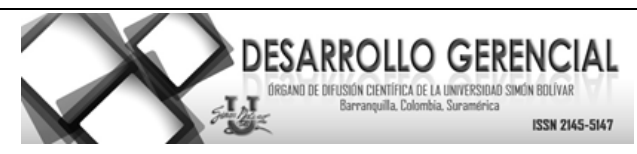

Adicionalmente es pertinente el diseño de programas de alfabetización de adultos, de fortalecimiento de las unidades económicas empresariales y capacitación para el trabajo de la población nativa, orientados fundamentalmente hacia actividades de protección y conservación del medio ambiente, así como de los recursos naturales de que dispone la ciénaga la Caimanera, y de esta manera promover el desarrollo de un ecoturismo sostenible.

\section{5.- REFERENCIAS}

Álvarez, C. (2009). Los determinantes sociales de la salud: más allá de los factores de riesgo. Revista Gerencia Política Salud. Bogotá, 8 (17), Extraido el 10 de noviembre de 2014 desde http:// www.scielo.org.co.

Aruj, R. (2008). Causas, consecuencias, efectos e impactos de las migraciones en Latinoamérica. Revista Papeles de la poblacion. Mexico, 55(14) 95-116. . Extraído el 12 de noviembre de 2014 desde http://www.redalyc.org.

Cepal. (2000). Equidad, desarrollo y ciudadanía. Una visión México. cepal.org. Extraído el 5 de octubre de 2014 desde http:// www.cepal.org.

MCIT (1996). Ley 300. Ley General del Turismo, Bogotá, Colombia: Imprenta Nacional.

MCIT (2012) Ley 1558 Nueva ley general del turismo, Bogotá, Colombia: Imprenta Nacional.

DANE 2. ( 2005 , de mayo). La visibilización de los grupos étnicos colombianos. Extraído el 14 de noviembre de 2014 desde http://www.dane.gov.co.

DANE. (2005). Censo2005.dane.gov.co. Extraído el 21 de marzo de 2014 desde http://www.dane.gov.co/index.php/poblacion-y-demografia/censos.

DANE. (2011). Actualización Censo/dane.gov.co. Extraído el 20 de marzo de 2014 desde http://www.dane.gov.co/.

DANE. (2014, 21 de marzo). Pobreza monetaria y multidimensional 2013. Extraído el 14 de agosto de 2014 desde http:http://www.dane.gov.co.

De Almeida, J. S. (2011). Ecoturismo científico en la planicie costera del extremo litoral sur del estado de São Paulo (Brasil). Estudios y perspectivas en turismo, 0(5),1196-1213.

Díaz de Iparraguirre, A. M. (2009). La Gestión compartida Universidad-Empresa en la formación del Capital Humano. Su relación con la competitividad y el desarrollo sostenible. Edición electrónica gratuita. Texto completo en www. eumed. net/tesis/2009/amdi. 


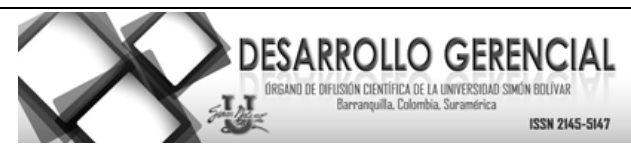

DNP. (2010). Documento regional para promover el desarrollo sostenible del golfo de Morrosquillo, isla de San Bernardo e isla Fuerte, Santiago de Tolú. Extraído el 11 de agosto de 2014 desde http://colaboracion.dnp.gov.co.

Méndez Heredia, G. (2008, 20 de octubre). Re-conceptualización del ecoturismo como factor de desarrollo humano integral como soporte de una propuesta de educación formal de postgrado. Extraído el 15 de octubre de 2014 desde http://repository.lasalle.edu.co.

Mincetur.gob.pe. (2006). Manual para la formulación del inventario de recursos turísticos a nivel nacional: Extraído el 10 de septiembre de 2014 desde http://www.mincetur.gob.pe.

Ministerio de Comercio, Industria y Turismo. (2003). Política Nacional de Ecoturismo. Extraído el 23 de noviembre de $2014 \quad$ desde http://cpps.dyndns.info/cpps-docsweb/planaccion/biblioteca/pordinario/Proceso\%20Ordinario/Tourism-

Recreation/PolticaNacionaldeEcoturismo.

Naranjo. (2002). Capacitación y formación profesional para jóvenes en Uruguay. Los programas opción joven y projóvenes para jóvenes en Uruguay. oitcinterfor.org. Extraído el 28 de octubre de 2013, desde http:// www.oitcinterfor.org/sites/default/files/file_publicacion/capfojo.pdf.

Ochoa, F. (2013). Visión comunitaria de los beneficios derivados del ecoturismo en el PNN AmacayacuAmazonas. Gestión y Ambiente, 16(1), 17-32.

OMT. (S. F.). Entender el turismo: Glosario Básico Extraído el el 10 de diciembre de 2014 desde http://media.unwto.org.

Organización Mundial del Turismo. (2003). Turismo y atenuación de la pobreza. España. Extraído el 14 de noviembre de 2014, desde http://www.edicionessimbioticas.info.

Ospina, M. \& Mora, R. (2013). Ecoturismo: diagnóstico y propuesta estratégica para la oferta de destinos ecoturísticos en Colombia por parte de las agencias de turismo localizadas en Bogotá, D.C. Revista Cuadernos Latinoamérica, 9(17), 9-31 Extraído 28 de Octubre 2013 desde http://issuu.com/universidadelbosque/docs/web_revista_cuadernos_latinoamerica/1?e=0/6598432.

Plan de Desarrollo Municipal. (2012). Alcaldía de Coveñas Sucre - Unidos es el Camino. covenassucre.gov.co. Extraído el 15 de julio de 2013 desde http://www.covenassucre.gov.co/index.shtml? $\mathrm{x}=1919601$.

Prada, L. (2009). El ecoturismo en el mundo contemporáneo: Una propuesta para Colombia. Bogota: Universidad Javeriana. 
Romero M., C. E. (2008). Informe sobre el estado de la biodiversidad en Colombia. Bogotá,: Instituto de Investigación Alexander von Humboldt.

Ross, S. (1999). Ecotourism: Towards congruence between theory and practice. Tourism Management, 20, 123-132.

Schkolnik, S. (2010). Acerca de la inclusión del enfoque de género en los censos de población y vivienda. Notas de Población, 91, 16.

Sen , A. (1999). Nuevo examen de la desigualdad . Madrid.: Alianza Editorial.

Silva, C. (2010). Modo de desarrollo humano: Realización de la libertad y búsqueda de la felicidad. Revista Desarrollo Gerencial, 2, 13-40. Extraído en julio de 2014 desde http://www.fuac.edu.co/recursos_web/observatorio/publicaciones/2014/Modo_desarrollo_humano .pdf.

Van der Duim, R. A. (2007). Humedales, reducción de la pobreza y desarrollo del turismo sostenible: oportunidades y limitaciones. Extraído el 14 de agosto de 2014. desde Wetlands Internationhttp://ramsar.conanp.gob.mx.

Vanegas, G. (2006). Ecoturismo, instrumento de desarrollo sostenible. Medellín: Universidad de Antioquia. Zamorano, F. (2008). Turismo alternativo. Servicios turísticos diferenciados: animación, turismo de aventura, turismo cultural, ecoturismo, turismo recreativo. México: Editorial Trillas. 